\title{
Economic Burden of Periprosthetic Joint Infection Following Primary Total Knee Replacement in a Developing Country
}

\author{
Faizan Iqbal, FCPS, Bilal Shafiq, FCPS, Syed Shahid Noor, FRCS, Zulfiqar Ali, FCPS, \\ Nouman Memon, FCPS, Naveed Memon, FCPS \\ Department of Orthopedic Surgery Liaquat National Hospital and Medical College, Karachi, Pakistan
}

Background: Periprosthetic joint infection is one of the devastating complications after primary total knee arthroplasty, which increases the financial burden on patients and affects their quality of life as well. The financial burden of periprosthetic joint infection after joint replacement in developed countries is well known. There is a need to evaluate the economic burden in developing countries such as Pakistan.

Methods: This is a single-center, retrospective, case-control study conducted at the Department of Orthopedic Surgery, Liaquat National Hospital Karachi. Cases of primary total knee arthroplasty performed during this study were divided into 2 groups: uneventful primary total knee arthroplasty and periprosthetic joint infection treated with 2-stage revision. To calculate the final cost, we divided the total hospital cost into the hospital stay cost and operating room cost.

Results: During study period, 32 patients were diagnosed with periprosthetic joint infection. The total cost of revision surgery for periprosthetic joint infection considering 2 hospitalizations was 1,780,222 \pm 313 ,686 Pakistani rupee (PKR). The total cost of uneventful arthroplasty was $390,172 \pm 51,460$ PKR. We observed significant difference with respect to economic details between the 2 groups.

Conclusions: Management of periprosthetic joint infection was 4.5 times more expensive than uneventful primary total knee arthroplasty. Measures should be undertaken to reduce the prevalence of periprosthetic joint infection, thereby reducing patients' economic burden.

Keywords: Total knee arthroplasty, Periprosthetic joint infection, Economic burden, Revision surgery

Periprosthetic joint infection (PJI) is one of the devastating complications after primary total knee arthroplasty (TKA), which increases the financial burden on patients and affects their quality of life as well. ${ }^{1-4)}$ Despite modern modalities for preventing infection, the incidence of PJI is $1 \%-2 \%$ after knee replacement surgery. ${ }^{5,6)}$ As the burden of the lower limb arthroplasty procedure is frequently in-

Received February 26, 2020; Accepted April 26, 2020

Correspondence to: Faizan Iqbal, FCPS

Liaquat National Hospital and Medical College, National Stadium Rd,

Sindh 74800, Karachi, Pakistan

Tel: +92-3402238126, Fax: +92-2134145034

E-mail:drfaizi1@yahoo.com creasing in the elderly population, the economic burden of PJI is steadily increasing as well. ${ }^{7)}$ The actual cost of treating patients with PJI is difficult to determine because it depends on multiple factors such as the specific type of treatment given to a patient, bacteria-specific antibiotics, and patient's comorbidities.

The spectrum of economic burden comprises inpatient cost as well as outpatient cost, which includes costs for follow-up visits, rehabilitation, and medication. PJI is a dilemma especially in developing countries such as Pakistan. According to the World Bank's classification, Pakistan belongs to the lower-middle-income group. ${ }^{8)}$ The management of PJI varies from an extended course of antibiotics to surgical debridement, which places massive 
financial burden on the health care system. The financial burden of PJI after joint replacement in developed countries is well known. ${ }^{9,10)}$

There is a need to evaluate the economic burden in developing countries such as Pakistan. The magnitude of problems is far greater in Pakistan than in well-developed countries because there is no health insurance system and all the expenses must be borne by patients themselves. This is the major difference between PJI treated in developing countries to that treated in well-developed countries. The rationale of this study is to evaluate the existing economic burden of treating infected knee joints and to provide cost-effective policies in order to minimize financial burden. The purpose of this study was to evaluate the in-hospital cost of treating infected joints after primary total knee replacement (TKR) and compare with the cost of uneventful TKA.

\section{METHODS}

This is a single-center, retrospective, case-control study conducted at the Department of Orthopedic Surgery, Liaquat National Hospital, which is one of the private hospitals located in Karachi, Pakistan. The study was approved by the Ethics Review Committee of the hospital (No. 0111-2018). Patients enrolled for the study were operated between January 2014 and December 2018. All patients in this study bore full expenses. Cases of TKA performed during this period were divided into 2 groups: uneventful primary TKA and PJI treated with 2-stage revision. The first group with uneventful arthroplasty served as a control. A total of 1,329 patients underwent primary TKA during this period in our hospital. Of those, 32 patients were diagnosed with PJI. Out of the 32 patients, 27 patients had primary TKA somewhere else, whereas 5 patients underwent primary knee joint replacement in our hospital. According to the Centers for Disease Control and Prevention, any sinus communicating with underlying prosthesis is considered prosthetic joint infection (PJI) until proven otherwise.

The inclusion criteria were all patients who underwent 2-stage revision surgery with a diagnosis of chronic PJI and patients who had uneventful primary TKA. Patients were excluded if they underwent debridement, antibiotics, and implant retention (DAIR) as in acute PJI or revision TKA due to aseptic reasons, if they had persistent infection despite surgical debridement and antibiotics and amputation was required as a last resort, or if they were readmitted due to any reasons including PJI after revision TKA.
Demographic data and clinical records of patients, which include the duration of stay in the orthopedic ward or intensive care unit, were retrieved from the hospital record. Administrative and economic data were retrieved by a non-medical person (AQ), who was blinded to patient diagnosis and outcome. Outpatient visits such as followup visits and rehabilitation cost were not included in this study. On admission, the medical conditions of all patients were assessed and classified according to the American Society of Anesthesiologists (ASA) grade. ${ }^{11)}$ All patients underwent 2-stage revision after being diagnosed with PJI. We used 3gm vancomycin as a cement spacer in all cases. Pus and deep tissue were sent for routine microbiological culture and sensitivity testing along with histological examination of the infected tissue. Tissue samples were obtained from 5 standard surgical sites such as synovium and medullary canal of the femur and tibia. ${ }^{12)}$ In patients with PJI, the causative organism and its antibiotic susceptibility and the date of diagnosis and treatment were retrieved from hospital records. An infectious disease team was kept on board. Patients took intravenous antibiotics for 6 weeks. Some patients required 2 antibiotics depending on organism susceptibility to a particular antibiotic. Reaspiration was performed 2 weeks after stopping antibiotics. Revision surgery was performed at 3 to 4 months after the first-stage procedure. To calculate the final cost, we divided the total hospital cost into 2 categories: hospital stay cost and operating room cost.

\section{Hospital Stay Cost}

Hospital stay cost includes (1) ward or intensive care unit (ICU) cost for maintenance, meals, and accommodation, (2) diagnostic test cost for imaging, microbiology, and blood tests, or (3) medication cost with or without antibiotics. The duration of stay in a ward and ICU was retrieved from hospital records. The diagnostic tests performed in the uneventful arthroplasty group include complete blood counts, urea creatinine and electrolytes, coagulation profile, urine microbiology culture and sensitivity, hepatitis B and C profile, X-rays (preoperative and postoperative), and standing scanogram. The diagnostic tests performed in the revision TKR group, considering the 2 hospitalizations, were also similar, with addition of C-reactive protein, erythrocyte sedimentation rate, cultures, and histopathology. Hepatitis profile was performed on the first admission in the revision cases.

\section{Operating Room Cost}

Operatinng room cost refers to costs of equipment and general and clinical support specific for surgery along 
Iqbal et al. Economic Burden of Periprosthetic Joint Infection in Pakistan

Clinics in Orthopedic Surgery • Vol. 12, No. 4, $2020 \bullet$ www.ecios.org

with surgeon and anesthetist fees. This includes the cost of operating room and recovery room charges, epidural and medications used by an anesthetist, and surgical drapes. Cost of clinical materials used such as cement spacer during the first-stage procedure and implants used during revision surgery such as constrained condylar knee, rotating hinge knee with or without metaphyseal sleeves, and metal augments or wedges were considered.

With all these variables in mind, the average cost for each of the differentiated groups is reported in Pakistani rupee, which is then converted to US dollar (\$). Costs of the implant and other consumable items remained the same throughout the study period. These prices were converted to US dollar with the market exchange rate at the end of the study period. A computer-generated algorithm was used to select patients with uneventful primary TKA. Demographic parameters such as age, sex, length of stay in ward or ICU, time in operating room, and ASA score were matched between the 2 groups of patients. Patients who did not meet abovementioned parameters were excluded from our study. If parameters were identical in more than 2 control patients, patients were selected using the computer-generated randomization algorithm. The data were analyzed statistically using statistics version 20.0. Categorical variables were compared through chi-square and Fisher exact test. Continuous variables were compared using Mann-Whitney test. Multivariable regression analysis was done to identify the variables that place major financial burden on patient economy. The level of significance was set at $p<0.05$.

\section{RESULTS}

During the study period, 32 patients were diagnosed with PJI. We were unable to find economic data in 3 patients and 2 patients were lost to follow-up after the first-stage procedure, so ultimately 27 patients were included in this study and served as cases. We obtained records of $27 \mathrm{pa}-$ tients who underwent primary arthroplasty during the study period and were considered as controls. Patients with uneventful primary TKA were selected randomly, whereas 27 patients who suffered PJI were evaluated in terms of economic burden. All patients with PJI underwent 2-stage revision surgery in this study. Basic demographic data and clinical data of the cohort are presented in Table 1. There was no statistical difference between cases and controls with respect to age, ASA score, and sex. However, we observed statistically significant difference between the 2 groups in terms of length of stay in ward and ICU and time in operating room $(p<0.05)$.
We also evaluated in-hospital economic data of 27 cases with PJI. Economic calculations are presented in Table 2. Individual costs of the first- and second-stage revision surgery are presented in Table 3 . The total cost of revision surgery for PJI considering the 2 hospitalizations was found to be $1,780,222 \pm 313,686$ rupees ( $\$ 12,277$ $\pm 2,163)$. The total cost of uneventful arthroplasty was found to be $390,172 \pm 51,460$ rupees $(\$ 2,690 \pm 354.9)$. The total cost was the sum of 2 variables: the cost of hospital stay and operating room cost. We observed statistically significant difference with respect to economic details between the 2 groups $(p<0.05)$. We further divided the ward stay into 2 variables: semi-private room and private room. The cost of semi-private room in our hospital was $6,800$ rupees/day ( $\$ 47)$, whereas the cost of private room was 8.900 rupees/day ( $\$ 65)$. The mean duration of stay of patients with uneventful arthroplasty was $5 \pm 1$ days, whereas it was $11 \pm 3$ days with revision TKR $(p<0.05)$. The diagnostic tests were further divided into blood test, imaging, and microbiology. The total cost for blood test including inflammatory markers was 12,290 rupees (\$85). Imaging or X-ray cost for a unilateral knee was 2,220 rupees (\$15), whereas the bilateral knee X-ray cost was 4,440

\begin{tabular}{|c|c|c|c|}
\hline Variable & $\begin{array}{l}\text { Primary arthroplasty } \\
\qquad(\mathrm{n}=27)\end{array}$ & $\begin{array}{l}\text { Prosthetic joint } \\
\text { infection }(n=27)\end{array}$ & $p$-value \\
\hline Sex & & & 0.08 \\
\hline Male & 17 & 17 & \\
\hline Female & 10 & 10 & \\
\hline Age (yr) & $58.4 \pm 4.9$ & $61.3 \pm 5.9$ & 0.08 \\
\hline $\begin{array}{l}\text { Length of stay } \\
\text { in ward (day) }\end{array}$ & $5 \pm 1$ & $11 \pm 3$ & $0.004^{*}$ \\
\hline $\begin{array}{l}\text { Length of stay } \\
\text { in ICU (day) }\end{array}$ & 0 & $2 \pm 1$ & $0.001^{*}$ \\
\hline ASA score & & & 0.08 \\
\hline
\end{tabular}

\begin{tabular}{cccc} 
Class 1 & & & \\
Class 2 & 5 & 5 & \\
Class 3 & 15 & 15 & \\
Class 4 & 7 & 7 & $0.003^{*}$ \\
$\begin{array}{c}\text { Time in operating } \\
\text { room (min) }\end{array}$ & $58 \pm 5.6$ & $118 \pm 8.9$ & \\
\hline
\end{tabular}

Values are presented as mean \pm standard deviation.

ICU: intensive care unit.

${ }^{*} p$-value $<0.05$ is considered significant. 
Table 2. In-Hospital Economic Data of 27 Periprosthetic Joint Infection Cases

$$
\begin{array}{cc}
\text { Primary arthroplasty }(n=27) \\
\text { (price in PKR for } 1 \text { primary TKR) }
\end{array}
$$

Prosthetic joint infection $(n=27)$ (price in PKR for 1 revision TKR including first- and second-stage treatments)

Hospital stay cost

$\begin{array}{lccc}\text { Ward cost } & 42,539 \pm 8,346(294 \pm 58) & 89,345 \pm 12,346(616 \pm 85.14) & 0.001 \\ \text { Diagnostic test } & 13,425 \pm 1,892(92.5 \pm 13) & 29,456 \pm 2,572(203 \pm 17.7) & 12,941 \pm 2,041(89.24 \pm 14.07) \\ \text { Medication excluding antibiotics } & 11,242 \pm 1,792(77.5 \pm 12.3) & 68,925 \pm 13,942(475 \pm 96.1) \\ \text { Antibiotics } & 9,245 \pm 636(63.35 \pm 4.24) & 26,789 \pm 12,623(185 \pm 87) \\ \text { ICU stay (day) } & 0 & 227,456 \pm 43,524(5,054.6 \pm 300.2) \\ \text { Total cost } & 76,511 \pm 12,666(527.6 \pm 87.35) & & 0.090 \\ \text { Operating room (OR) cost } & 58,247 \pm 4,223(3,160 \pm 29.12) & 98,394 \pm 9,286(678.5 \pm 64.04) \\ \text { OR charge (epidural + medications) } & & 0.001 \\ \text { Clinical materials including implant } & 194,325 \pm 12,326 & 1,374,763 \pm 242,567(9,481 \pm 1,672)\end{array}$

TC3 fixed bearing

TC3 fixed bearing

$498,000 \pm 22,347$

TC3 with MBT

$998,000 \pm 24,786$

RHK

$1,610,000 \pm 74,269$

0.000

Metaphyseal augment

Surgeon fee

$93,000 \pm 11,000$

Anesthesia fee

$33,425 \pm 7,524(230 \pm 51.9)$

$54,623 \pm 12,897(376 \pm 88.94)$

0.002

um of OR charge + average cost of

$19,644 \pm 1,987(135.47 \pm 13.7)$

24,986 \pm 5,412 (172.3 \pm 37.32$)$

0.003

implant

$313,661 \pm 38,794(2,163 \pm 267.5)$

$1,552,766 \pm 270,162(10,708 \pm 1,856)$

0.001

Total cost (hospital stay cost + OR cost)

$390,172 \pm 51,460(2,690 \pm 354.9)$

$1,780,222 \pm 313,686(12,277 \pm 2,163)$

0.002

Values are presented as mean \pm standard deviation. Values in parentheses are in dollars.

PKR: Pakistani rupee, TKR: total knee replacement, ICU: intensive care unit, PS: posterior-substituting, CR: cruciate-substituting, TC3: total condylar, MBT: mobile-bearing tray, RHK: rotating hinge knee.

${ }^{*} p$-value $<0.05$ is considered significant.

rupees (\$31). The cost for microbiology (histopathology and cultures) was 5,000 rupees (\$35). Diagnostic tests were also found to be statistically significant in both groups ( $p$ $<0.05$ ). Intravenous antibiotics were given for 6 weeks according to culture and sensitivity. We mostly encountered methicillin-resistant staphylococcus aureus (MRSA), methicillin-sensitive staphylococcus aureus (MSSA), pseudomonas, and streptococcus during the study period. We used vancomycin for MRSA, Amoxiclav for MSSA, Piperacillin/Tazobactam for pseudomonas, and penicillin or Cephalosporins for streptococcus. One vial of vancomycin costs 1,331 rupees (\$9), tazocin 870 rupees (\$6), amoxiclav 670 rupees (\$4.6), and cephalosporin 165 rupees $(\$ 1.2)$. Organisms and their antibiotics sensitivity are presented in Table 3. The total cost of stay in ICU in our hospital was 13,500 rupees/day (\$89.66). None of the patients with primary arthroplasty stayed at ICU, whereas patients with PJI had a mean ICU stay of $3 \pm 1$ days $(p<0.05)$. The cost of operating room was 15,500 rupees $(\$ 107)$, recovery room 10,000 rupees (\$69), epidural kit 15,000 rupees $(\$ 107)$, surgical drapes 7,500 rupees (\$52), and medications used by anesthetist was 10,000 rupees $(\$ 69)$. The average OR 
Iqbal et al. Economic Burden of Periprosthetic Joint Infection in Pakistan

Clinics in Orthopedic Surgery • Vol. 12, No. 4, $2020 \bullet$ www.ecios.org

Table 3. Individual Cost for the First- and Second-Stage Revision

Variable
First-stage surgery (including cost of implant removal and cement spacer insertion)
Second-stage surgery (including cost of revision TKA)
Total cost

Hospital stay cost

\begin{tabular}{|c|c|c|c|}
\hline Ward cost & $32,425 \pm 4,565(224 \pm 31.48)$ & $56,920 \pm 7,781(392.5 \pm 142)$ & $89,345 \pm 12,346(616 \pm 85.14)$ \\
\hline Diagnostic test & $13,966 \pm 1,196(96.3 \pm 8.25)$ & $15,490 \pm 1,376(107 \pm 9.5)$ & $29,456 \pm 2,572(203 \pm 17.7)$ \\
\hline Medications excluding antibiotics & $5,435 \pm 925(37.48 \pm 6.4)$ & $7,506 \pm 1,116(52 \pm 8)$ & $12,941 \pm 2,041(89.24 \pm 14.07)$ \\
\hline Antibiotics & $48,475 \pm 7,879(334.5 \pm 54.3)$ & $20,450 \pm 6,063(141 \pm 41.8)$ & $68,925 \pm 13,942(475 \pm 96.1)$ \\
\hline ICU stay & $12,565 \pm 7,245(86.65 \pm 50)$ & $14,224 \pm 5,378(98.1 \pm 37.1)$ & $26,789 \pm 12,623(185 \pm 87)$ \\
\hline Total cost & $112,866 \pm 21,810(778 \pm 150)$ & $114,590 \pm 21,714(790 \pm 150)$ & $227,456 \pm 43,524(5,054.6 \pm 300.2)$ \\
\hline \multicolumn{4}{|l|}{ Operating room (OR) cost } \\
\hline OR charge (epidural plus medications) & $45,435 \pm 3,065(313 \pm 21.13)$ & $52,959 \pm 6,221(365 \pm 42.9)$ & $98,394 \pm 9,286(678.5 \pm 64.04)$ \\
\hline \multicolumn{4}{|l|}{ Clinical materials including implant } \\
\hline Average cost & $11,545 \pm 3,897(79.6 \pm 26.9)^{*}$ & $1,363,218 \pm 238,670(9,401 \pm 1,645)$ & $1,374,763 \pm 242,567(9,481 \pm 1,672)$ \\
\hline Surgeon fee & $15,275 \pm 4,871(105.4 \pm 33.5)$ & $39,348 \pm 8,026(271.4 \pm 55.2)$ & $54,623 \pm 12,897(376 \pm 88.94)$ \\
\hline Anesthesia fee & $11,235 \pm 1,235(77.5 \pm 8.5)$ & $13,751 \pm 4,177(94.8 \pm 28.8)$ & $24,986 \pm 5,412(172.3 \pm 37.32)$ \\
\hline $\begin{array}{l}\text { Sum of OR charge }+ \text { average cost of } \\
\text { implant }\end{array}$ & $83,490 \pm 13,068(575.6 \pm 90.1)$ & $1,469,276 \pm 257,094(10,133 \pm 1,773)$ & ) 1,552,766 $\pm 270,162(10,708 \pm 1,856)$ \\
\hline
\end{tabular}

Values are presented as mean \pm standard deviation. Values in parentheses are in dollars.

TKA: total knee arthroplasty, ICU: intensive care unit.

${ }^{*}$ Cost of cement used in antibiotic cement spacer.

cost after uneventful primary arthroplasty was 58,247 \pm 4,223 rupees $(\$ 3,160 \pm 29.12)$, whereas it was $98,394 \pm$ 9,286 rupees $(\$ 6,785 \pm 64.04)$ after revision TKR $(p<0.05)$. The average cost of implants used during primary TKA was $202,345 \pm 12,734$ rupees $(\$ 1,395.5 \pm 88)$, whereas it was $1,374,763 \pm 242,567$ rupees $(\$ 9,481 \pm 1,672)$ after revision knee replacement. The cost of specific implant used during revision surgery is presented in Table 2. Surgeon and anesthetist fees for primary and revision knee arthroplasty cases are also shown in Tables 2 and 3.

Multivariable regression analysis was done to identify the variables that place a major financial burden on patient economy. We found that the ward cost, diagnostic tests, antibiotics, clinical materials, and surgeon and anesthetist fees had major impact on the patient economy after diagnosis of PJI. The detailed results are shown in Table 4. Results showed that the burden of PJI was 4.5 times more as compared to uneventful primary TKA. These results will actually bring knowledge to health care professionals about the impact of PJI on patient economy. To minimize this burden, measures should be undertaken to improve our infection prevention strategies.

\section{Table 4. Multivariable Analysis}

\begin{tabular}{|c|c|c|}
\hline Variable & Odds ratio (95\% Cl) & $p$-value \\
\hline Ward cost & $9.452(1.020-1.462)$ & 0.004 \\
\hline Diagnostic test & $4.824(1.069-1.331)$ & 0.001 \\
\hline Medications excluding antibiotics & $1.283(1.248-1.756)$ & 0.064 \\
\hline Antibiotics & $19.826(0.842-1.214)$ & $0.014^{*}$ \\
\hline ICU stay & $2.142(1.123-1.134)$ & 0.031 \\
\hline $\mathrm{OR}$ cost & $1.183(1.148-1.556)$ & 0.064 \\
\hline Clinical materials including prosthesis & $17.826(0.842-1.214)$ & $0.011^{*}$ \\
\hline
\end{tabular}

$\mathrm{Cl}$ : confidence interval, ICU: intensive care unit, OR: operating room. ${ }^{*} p$-value $<0.05$ is considered significant.

\section{DISCUSSION}

It is with no surprise that management of PJI represents a massive economic burden on health care systems, hospitals, and patients. There are only few papers available that address this issue in well-developed countries, but there is a need to evaluate the impact of management of PJI on 
patient economy in developing countries such as Pakistan. To the best of our knowledge, this report is the first from a single center of Karachi, Pakistan, regarding the economic burden of PJI following primary TKR. The total financial burden of management of PJI on patient economy is much more than the sum of all available data that can be objectively ascertained. In the current study, we only reported the in-hospital cost and we were unable to report the outpatient cost such as follow-up visits and rehabilitation cost. We were also unable to assess the indirect cost such as productivity loss and absenteeism from work of patients and their caregivers. It was also very difficult to compare our economic burden after management of PJI with that of other developing countries due to differences in the healthcare system and economic standard of particular nations.

Kapadia et al. ${ }^{13)}$ in their study compared 21 infected primary TKR with 21 non-infected patients who underwent uneventful TKR. They found that patients with PJI had significantly prolonged hospitalizations. The total cost for patients with PJI was $\$ 116,383$ (range, $\$ 44,416-\$ 269,914)$, which was significantly greater than uneventful primary knee arthroplasty with \$28,249 (range, $\$ 20,454-\$ 47,957)$. This study was conducted in Sinai Hospital, which is one of the renowned private American hospitals. We were unable to compare our economic burden with the results presented by Kapadia et al. ${ }^{13)}$ due to the difference in health care system and economic standard between 2 countries. In our study, patients with PJI had more prolonged stay as well considering 2 hospitalizations, which actually imposed a major impact on the patients and their family economy $(p<0.05)$ as shown in Tables 1 and 4. In our study, ICU care was decided on the basis of ASA score and postoperative evaluation in recovery room. Twenty-two patients (81.4\%) with PJI had ASA scores of 3 and 4 and warranted ICU admission for further monitoring as per decided by the anesthesia team. Patients with uneventful arthroplasty had ASA scores of 1 and 2 and did not require ICU admission postoperatively.

Garrido-Gomez et al. ${ }^{14)}$ performed a descriptive analysis of economic cost of patients with PJI in the public health system of Andalusia, Spain. They presented results of PJI based on occurrence. The mean cost per patient was $\$ 24,980(€ 19,270.80)$ for patients with early PJI and rose to $\$ 78,111(€ 60,257)$ for those with late PJI. Our study excluded patients who presented early and underwent the DAIR procedure. They also concluded that hospital stay followed by the cost of surgical implants puts major impact on patient economy. Fernandez-Fairen et al. ${ }^{15)}$ performed a systematic review and found that revision knee arthro- plasty was 2 to 4 times more expensive than uneventful primary knee arthroplasty. We found that management of PJI was 4.5 times more expensive than primary uneventful knee arthroplasty. We also observed that the ward cost, diagnostic test, antibiotics, clinical materials including prosthesis, and surgeon and anesthetist fees used during revision surgery had significant impact on the economic status of patients who presented with PJI as shown in Table 4 .

The economic burden of PJI can be reduced by making health policies as well as implementing preventive measures to attenuate the risk of PJI. Knowledge of the cost related to PJI is necessary to optimize existing health resources in developing countries such as Pakistan. Although treatment protocols and guidelines exist to prevent the incidence of PJI following a joint replacement procedure, a more proactive, individualized approach may be necessary to sort out this issue. ${ }^{16,17)}$ There is a need to identify highrisk patients with proactive implementation of pre- and postoperative protocols in order to prevent this devastating complication, thereby reducing financial burden. Previous studies reported a number of evidence-based protocols, which have proven to be effective in reducing PJI such as the use of prophylactic antibiotics and negative pressure wound therapy on surgical incisions. ${ }^{18,19)}$ According to data from OECD (organization for economic cooperation and development), the U.S. spent $17.8 \%$ of its gross domestic product (GDP) on health care, while the average spending level among all high-income countries was $11.5 \%$ of $\mathrm{GDP}^{20)}$ On the other hand, the share of total public health expenditure in Pakistan as percentage of GDP is only $0.7 \%$. Basically, PJI is the dilemma of not only developing countries but also well-developed countries. Well-developed countries cope with this issue well because of their health insurance and existing data will be sufficient to make necessary actions to prevent this devastating complication of primary TKA. On other hand, Pakistan is a developing country, patients have no health insurance, and we have insufficient data to make necessary steps. Thus, the study was conducted to highlight the poor picture of health expenditures in Pakistan. The increase in the expenditure as percentage of GDP on health besides other social expenditures should be strongly emphasized.

The major limitation of this study is that we mainly focused on the in-hospital cost and excluded outpatient cost such as follow-up visits and rehabilitation cost so that the exact economic burden could not be determined. Another drawback of the study is that it was a single-center study and analysis was performed only in patients who underwent 2-stage revision surgery although this small, retrospective, case-control study demonstrated some important 
Iqbal et al. Economic Burden of Periprosthetic Joint Infection in Pakistan

Clinics in Orthopedic Surgery • Vol. 12, No. 4, $2020 \bullet$ www.ecios.org

conclusions. The cost of PJI treatment was $\$ 12,277 \pm$ 2,163 , which was 4.5 times higher than uneventful primary TKA. The findings of this study show that PJI following TKR imposes a major economic burden on patients.

\section{CONFLICT OF INTEREST}

No potential conflict of interest relevant to this article was reported.

\section{ACKNOWLEDGEMENTS}

We appreciate contribution of Ali Qureshi (Secretary, Department of Orthopedic Surgery, Liaquat National Hospital) for this study.

\section{REFERENCES}

1. Whitehouse JD, Friedman ND, Kirkland KB, Richardson WJ, Sexton DJ. The impact of surgical-site infections following orthopedic surgery at a community hospital and a university hospital: adverse quality of life, excess length of stay, and extra cost. Infect Control Hosp Epidemiol. 2002;23(4):183-9.

2. Iqbal F, Younus S, Asmatullah, Zia OB, Khan N. Surgical site infection following fixation of acetabular fractures. Hip Pelvis. 2017;29(3):176-81.

3. Patel A, Pavlou G, Mujica-Mota RE, Toms AD. The epidemiology of revision total knee and hip arthroplasty in England and Wales: a comparative analysis with projections for the United States: a study using the National Joint Registry dataset. Bone Joint J. 2015;97(8):1076-81.

4. de Lissovoy G, Fraeman K, Hutchins V, Murphy D, Song D, Vaughn BB. Surgical site infection: incidence and impact on hospital utilization and treatment costs. Am J Infect Control. 2009;37(5):387-97.

5. Horan TC, Andrus M, Dudeck MA. CDC/NHSN surveillance definition of health care-associated infection and criteria for specific types of infections in the acute care setting. Am J Infect Control. 2008;36(5):309-32.

6. Dale H, Fenstad AM, Hallan G, et al. Increasing risk of prosthetic joint infection after total hip arthroplasty. Acta Orthop. 2012;83(5):449-58.

7. Sukeik M, Haddad FS. Periprosthetic joint infections after total hip replacement: an algorithmic approach. SICOT J. 2019;5:5.

8. World Bank. Gross national income country classification. Washington: World Bank; 2013.

9. Peel TN, Dowsey MM, Buising KL, Liew D, Choong PF. Cost analysis of debridement and retention for management of prosthetic joint infection. Clin Microbiol Infect. 2013;19(2):181-6.

10. Kurtz SM, Lau E, Watson H, Schmier JK, Parvizi J. Economic burden of periprosthetic joint infection in the United States. J Arthroplasty. 2012;27(8 Suppl):61-5.e1.
11. Daabiss M. American Society of Anaesthesiologists physical status classification. Indian J Anaesth. 2011;55(2):111-5.

12. Arduino JM, Kaye KS, Reed SD, et al. Staphylococcus aureus infections following knee and hip prosthesis insertion procedures. Antimicrob Resist Infect Control. 2015;4:13.

13. Kapadia BH, McElroy MJ, Issa K, Johnson AJ, Bozic KJ, Mont MA. The economic impact of periprosthetic infections following total knee arthroplasty at a specialized tertiary-care center. J Arthroplasty. 2014;29(5):929-32.

14. Garrido-Gomez J, Arrabal-Polo MA, Giron-Prieto MS, Cabello-Salas J, Torres-Barroso J, Parra-Ruiz J. Descriptive analysis of the economic costs of periprosthetic joint infection of the knee for the public health system of Andalusia. J Arthroplasty. 2013;28(7):1057-60.

15. Fernandez-Fairen M, Torres A, Menzie A, et al. Economical analysis on prophylaxis, diagnosis, and treatment of periprosthetic infections. Open Orthop J. 2013;7:227-42.

16. Berrios-Torres SI, Umscheid CA, Bratzler DW, et al. Centers for Disease Control and Prevention Guideline for the Prevention of Surgical Site Infection, 2017. JAMA Surg. 2017;152(8):784-91.

17. Allegranzi B, Bischoff $\mathrm{P}$, de Jonge $\mathrm{S}$, et al. New WHO recommendations on preoperative measures for surgical site infection prevention: an evidence-based global perspective. Lancet Infect Dis. 2016;16(12):e276-87.

18. Porucznik MA. "Zero in on zero" to reduce complications: orthopaedic surgeon leads way to improving care in joint replacement. Rosemont: AAOS Now; 2011.

19. Karlakki SL, Hamad AK, Whittall C, Graham NM, Banerjee $\mathrm{RD}$, Kuiper JH. Incisional negative pressure wound therapy dressings (iNPWTd) in routine primary hip and knee arthroplasties: a randomised controlled trial. Bone Joint Res. 2016;5(8):328-37.

20. Papanicolas I, Woskie LR, Jha AK. Health care spending in the United States and other high-income countries. JAMA. 2018;319(10):1024-39. 